\title{
Linx
}

Revue des linguistes de l'université Paris X Nanterre

$80 \mid 2020$

L'héritage de Jean Dubois et Françoise Dubois-Charlier

\section{Liste des publications de Jean Dubois}

\section{(2) OpenEdition}

1 Journals

Édition électronique

URL : http://journals.openedition.org/linx/6621

DOI : $10.4000 /$ linx.6621

ISSN : 2118-9692

Éditeur

Presses universitaires de Paris Nanterre

Référence électronique

"Liste des publications de Jean Dubois », Linx [En ligne], 80 | 2020, mis en ligne le 10 juillet 2020, consulté le 05 août 2020. URL : http://journals.openedition.org/linx/6621

Ce document a été généré automatiquement le 5 août 2020.

Département de Sciences du langage, Université Paris Ouest 


\section{Liste des publications de Jean Dubois}

1 Les trois premières parties de cette liste (y compris dans leur mise en page) ont été établies par Jean Dubois et Françoise Dubois-Charlier eux-mêmes. La quatrième partie, élaborée après eux, procure notamment les références d'accès à de nouvelles versions de leurs deux dictionnaires informatisés, LVF (Les Verbes Français) et le DEM (Dictionnaire Electronique des Mots).

\section{Linguistique française.}

\section{a. Ouvrages.}

2 Dictionnaire de la langue française classique, en collaboration avec René Lagane, préface de P. Clarac, avec index grammatical, Paris, Librairie classique Belin, 1960, 508 pages, repris sous le titre Dictionnaire du français classique, en collaboration avec Alain Lerond, Paris, Larousse, 1971, 511 pages, réédité en 1988, 2001, 509 pages (sans index grammatical).

3 Le vocabulaire politique et social en France de 1869 à 1872 à travers les œuvres des écrivains, les revues et les journaux. Thèse pour le doctorat ès lettres, Paris, Larousse, Paris 1962, 460 pages.

4 Etude sur la dérivation suffixale en français moderne et contemporain. Essai d'interprétation des mouvements observés dans le domaine de la morphologie des mots construits. Thèse complémentaire pour le doctorat ès lettres, Paris, Larousse, Paris 1962, 118 pages.

5 Nouveau dictionnaire étymologique et historique, avec Henri Mitterand (refonte du Dictionnaire étymologique de A. Dauzat), Paris, Larousse, 1964, 805 pages, réédité et corrigé en 1981, 1991, 1993, 2001.

Grammaire structurale : nom et pronom, Paris, Larousse, 1965, 192 pages.

7 Grammaire structurale : le verbe, Paris, Larousse, 1965, 216 pages. 

Jacqueline Casalis, Henri Meschonnic, Paris, Larousse, 1967, 1224 pages, devenu en 1980 Dictionnaire du français contemporain illustré (DFC), 1263 pages.

Grammaire structurale : la phrase et les transformations, Paris, Larousse, 1969, 187 pages.

Eléments de linguistique française, avec Françoise Dubois-Charlier, Paris, Larousse, 1970, 295 pages.

Introduction à la lexicographie : le dictionnaire, avec Claude Dubois, Paris, Larousse, 1971, 208 pages.

La nouvelle grammaire du français, avec René Lagane, Paris, Larousse, 1973, 266 pages, réédité en 1988.

Dictionnaire de linguistique, avec Mathée Giacomo, Louis Guespin, Christiane Marcellesi, Jean-Baptiste Marcellesi, Jean-Pierre Mevel, Paris, Larousse, 1973, 516 pages, réédité en 1994.

Lexis, dictionnaire de la langue française, sous la direction de J. Dubois, avec Cl. Kannas, J. P. Mevel, S. Hudelot, etc., Paris, Larousse, 1975, 1950 pages. ; réédité et augmenté sous le nom de Lexis, Larousse de la langue française, 1979, 2109 pages ; réédité en 2002.

Grammaire de base, Paris, Larousse, 1976, 78 pages.

Dictionnaire du français langue étrangère Niveau 1, avec F. Dubois-Charlier, Paris, Larousse, 1978, 910 pages.

Dictionnaire du français langue étrangère Niveau 2, avec F. Dubois-Charlier, Paris, Larousse, 1979, 1088 pages.

Larousse de l'orthographe, avec F. Dubois-Charlier, Paris, Larousse, 1982, 176 pages, devenu Nouveau Larousse de l'orthographe en 1994.

Dictionnaire du français au collège, refonte du DFC, sur des bases alphabétiques, insertion de la phonétique des entrées, Paris, Larousse, 1986, 1060 pages.

Petit dictionnaire de la langue française, (texte du DFC illustré), collection Références, Paris, Larousse, 1988, 1095 pages.

Dictionnaire de poche de la langue française, avec F. Dubois-Charlier, J.P. Mevel, Paris, Hachette, 1993, 620 pages.

Dictionnaire électronique des verbes français, avec F. Dubois-Charlier, 1993, remanié en 1994, 25.610 entrées ; contrat avec Cora, 1996, sur Internet ${ }^{1}$.

Dictionnaire électronique des verbes français, avec F. Dubois-Charlier, 1993, 276 pages, transcription de la version électronique de 1993.

Les verbes français (d'après le "Dictionnaire électronique des Verbes"), avec F. DuboisCharlier, Paris, Larousse, 1994, 458 pages.

Dictionnaire électronique des mots français, avec F. Dubois-Charlier, 136.867 entrées, Aix, $1995^{2}$.

Dictionnaire électronique des affixes (préfixes et suffixes), avec F. Dubois-Charlier, 4290 entrées, Aix, 1995.

Dictionnaire électronique des locutions, avec F. Dubois-Charlier, 5810 entrées, Aix, $1995^{3}$.

La dérivation suffixale, avec F. Dubois-Charlier, Paris, Nathan, 1999, 320 pages. 
Dictionnaire des suffixes en français, avec F. Dubois-Charlier, Aix, 2000, 865 pages (disponible prochainement sur le site de MoDyCo)

Composition et préfixation, avec F. Dubois-Charlier, Aix, 2001, 312 pages (disponible prochainement sur le site de MoDyCo)

Structures verbales, avec F. Dubois-Charlier, Aix, 2002, 303 pages.

Locutions en français, avec F. Dubois-Charlier, Aix, 2004, 414 pages (disponible prochainement sur le site de MoDyCo)

Adjectifs en français, avec F. Dubois-Charlier, Aix, 2005, 303 pages (disponible prochainement sur le site de MoDyCo)

Dictionnaire étymologique et historique du français, refonte totale du Nouveau Dictionnaire étymologique (encadrés, index alphabétique, etc.), avec H. Mitterand et la collaboration de F. Dubois-Charlier, Paris, Larousse, 2005.

\section{b. Articles.}

"Les notions d'unité sémantique complexe et de neutralisation dans le lexique", Cahiers de Lexicologie, Didier, 1960, 2, pp. 62-66.

"Le mouvement général du vocabulaire français de 1949 à 1960 d'après un dictionnaire d'usage", avec L. Guilbert, H. Mitterand et J. Pignon, Le Français moderne, juillet 1960, pp. 86-107, 196-211. Repris dans Introduction à la lexicographie, Paris, Larousse, 1971, pp. 111- 132.

"La notion de degré dans le système morphologique du français moderne", avec L. Guilbert, Journal de psychologie, mars 1961, pp. 57-64.

"Formation du système préfixal intensif en français moderne et contemporain", avec L. Guilbert, Le Français moderne, avril 1961, pp. 87-112.

"Le 'néo-français', réalité ou illusion?", La Pensée, 1961, avril, pp. 52-67. Traduit en allemand dans Die französchische Sprache von heute, Buchgesellschaft, 1983, pp. 145-166.

"Problèmes de méthode en lexicologie. Les notions d'unité sémantique complexe et de neutralisation dans le lexique", Cahiers de lexicologie, II, Paris, Didier, 1961, 62-67.

"Recherches lexicographiques. Esquisse d'un dictionnaire structural", Etudes de linguistique appliquée, Paris, Didier, 1962, 1, pp. 43-44.

2 "Espardre et espartir : conflit homonymique", avec R. Lagane, Le Français moderne, 1963, avril, pp. 105-110.

"L'emprunt en français", L'information littéraire, 1963, I, pp. 8-11.

"Recherches sur l'intonation française. La représentation segmentale et les réalisations graphiques de l'intonation en français contemporain", Etudes de linguistique appliquée, 1963, II, pp. 154, Paris, Didier.

"Distribution, ensemble et marque dans le lexique", Cahiers de lexicologie, 1964, 4, pp. 5-16.

"Représentation des systèmes paradigmatiques formalisés dans un dictionnaire structural", Cahiers de Lexicologie, 1964, 5, pp 3-15. 
"La traduction de l'aspect et du temps dans le code français (structure du verbe)", Le Français moderne, 1964, janvier, pp. 1-26.

"Terminologie linguistique", avec P. Marcie, Le Français moderne, 1964, juillet, pp. 11-21, et la suite dans Le Français moderne, 1965, janvier, pp. 21-33. 4

"Transformation négative et organisation des classes lexicales", avec L. Irigaray et P. Marcie, Cahiers de lexicologie, 1965, 7, pp. 3-42.

"Grammaire transformationnelle et morphologie", Le Français moderne, 1965, avril, pp. 81- 96, et juillet, pp. 178-187.

"Essai lexico-statistique historique du français contemporain - les mots commençant par h", La linguistique, 1965, 2, pp. 103-115.

"Les problèmes du vocabulaire technique", Cahiers de lexicologie, 1966, 9, pp. 104-112.

"Résolution des polysémies dans les textes écrits et structuration de l'énoncé", Actes du 1 er colloque de linguistique appliquée (Nancy 1962), Klincksieck, 1966.

"Essai d'analyse distributionnelle", Le Français moderne, 1966, juillet, pp. 185-209.

"Propriétés des phrases non-achevées en français", Bulletin de la Société de Linguistique, 1966, tome LXI, pp. 110-127.

"Problèmes de linguistique transformationnelle. Modèles précorrecteurs d'erreurs dans la transformation passive", Journal de psychologie, 1966, 4, pp. 29-55.

"Utilisation des statistiques lexicographiques pour l'étude structurale du lexique", dans Statistique et analyse linguistique, Paris, P.U.F., 1966, pp. 95-98.

"Pourquoi des dictionnaires ?" Information sur les sciences sociales, VI, 4, Mouton, 1967, pp. 101-112.

"Structuralisme en linguistique," La Pensée, 1967, 135, pp. 19-28.

"Production de phrases dans la population d'âge scolaire", avec G. Assal, A.M. Ramier, Journal de Psychologie, 1968, 2, pp. 183-207.

"Linguistique et révolution", avec J. Sumpf, Communications, 1968, 12, pp. 148-158.

"Lexicologie et analyse d'énoncé", Cahiers de lexicologie, 1969, 15, pp. 115-126.

"Grammaire distributionnelle", Langue française, 1969, 1, pp. 41-48.

"Grammaire générative et transformationnelle", Langue française, 1969, 1, pp. 49-57.

"Problèmes de l'analyse du discours", Langages, 1969, 13, pp. 3-7.

"Enoncé et énonciation", Langages, 1969, 13, pp. 100-110.

"Principes et méthode de l'analyse distributionnelle", avec F. Dubois-Charlier, Langages, 1970, 20, pp. 3-13.

"Un modèle d'enseignement $d u$ français : analyse linguistique des rapports d'agrégation et du CAPES", avec J. Sumpf, Langue française, 1970, 5, pp. 27-44.

"Les dossiers scolaires : étude linguistique", avec J. Sumpf, O. Motteau, Langue française, 1970, 5, pp. 86-93.

"L'orthographe", avec J. Sumpf, D. Meyrat, Langue française, 1970, 5, pp. 100-117.

"Analyse linguistique des rédactions", avec D. Bouix, B. Soulié, J. Sumpf, Langue française, 1970, 5, pp. 118-126. 
"Dictionnaire et discours didactique", Langages, 19, 1970, pp. 35-47, traduit en allemand dans Probleme des Wörterbuchs, Wissenschafdiche Buchgesellchaft, Darmstadt, 1985.

"Les études de grammaire et de vocabulaire français de Georges Gougenheim", Journal de Psychologie, juin 1971, pp. 207-211.

"Avant-propos", pp. 15-19 (sur l'analyse du discours) dans Le Congrès de Tours, par J.-B. Marcellesi, Paris, Le Pavillon, 1971, 357 pages.

"Grammaire scientifique et grammaire pédagogique", Langue française, 1972, 14, pp. 6-31,

"Le passif", avec F. Dubois-Charlier, Journal de psychologie, 1973, 2, pp. 79-92.

"Dictionnaire et syntaxe", Lexique, 1983, 2, pp. 85-88. Lille, P.U.L..Actes du colloque franco-néerlandais des 28-29 avril 1981 à Amsterdam.

"Pour relancer le débat. Lexicographie et grammaire - entretien avec J. Dubois", Le français dans le monde, Recherches et applications, 1989, Février-Mars, pp. 199-208

"Dictionnaire électronique du verbe français", avec F. Dubois-Charlier, communication dans Actes du IXe congrès européen sur la grammaire et le lexique des langues romanes, 1989.

"Le genre dans les noms d'animaux", LINX, 1989, 21, pp. 87-91.

"Incomparabilité des dictionnaires", avec F. Dubois-Charlier, Langue française, 1990, 87, pp. 5-10.

"A propos des dictionnaires électroniques des verbes", Travaux de linguistique, 1991, 23, pp. 141-142.

3 "Dictionnaire électronique des verbes français", Mélanges d'Alain Lerond - Etudes de linguistique française à la mémoire d'A. Lerond", numéro spécial de Linx, 1991, pp. 213-230.

"Les collectifs d'êtres vivants", avec F. Dubois-Charlier, Linx, 1996, pp. 125-132.

"Synonymie syntaxique et classification des verbes français", avec F. Dubois-Charlier, Langages, 1997, 128, pp. 51-71.

"La combinatoire lexico-syntaxique dans le Dictionnaire électronique des mots. Les termes de musique à titre d'illustration", avec F. Dubois-Charlier, Langages, 2010, 179-180, pp. 31-56.

\section{Neurolinguistique.}

\section{a. Ouvrage.}

37 La naissance de la neuropsychologie du langage (1825-1865), avec H. Hécaen, Paris, Flammarion, 1969, 274 pages.

\section{b. Articles.}

88 "Aspects du fonctionnement du code linguistique chez les aphasiques moteurs", avec D. Cohen, M. Gautier, H. Hécaen et R. Angelergues, Neuropsychologia, 1963, 1, pp. 165-177.

"Etude neurolinguistique de l'aphasie de conduction", avec H.Hécaen, R. Angelergues, A. Maufras du Chatellier et P. Marcie, Neuropsychologia, 1964, 2, pp. 9-44. 


\section{R. L'Hermit 315 - 331.} 31-40.

"Approche d'une neurolinguistique", Etudes de linguistique appliquée, 1964, 3, pp. 3-11.

"Comparaison neurolinguistique et neuropsychologique de deux observations anatomocliniques d'aphasie", avec H. Hécaen, R. Angelergues, C. Vedrennes et P. Marcie, Revue Neurologique, 3, 1964, pp. 401-411.

"Analyse distributionnelle en neurolinguistique : le comportement verbal des aphasiques dans les épreuves de langage répété", avec P. Marcie, L. Irigaray, H. Hécaen, Langage et comportement, 1965, 1, pp. 111-134.

"Les réalisations du langage chez les malades atteints de lésions de l'hémisphère droit", avec P. Marcie, H. Hécaen et R. Angelergues, Neuropsychologia, 1965, 3, pp. 217-245.

"Approche linguistique des aphasies", avec H. Hécaen et R. Angelergues, Bulletin de psychologie, 1966, 247, XIX, pp. 8-12.

"Les structures linguistiques de la parenté et leurs perturbations dans les cas de démence et de schizophrénie", avec L. Irigaray, Cahiers de Lexicologie, 1966, 8, pp. 47-69.

"Le problème de l'aphasie des polyglottes : remarques sur quelques observations", avec "Etude des performances aux épreuves linguistiques des sujets atteints de syndromes parkinsoniens", avec H. Hécaen, P. Marcie, G. Mazars, Encéphale, 1966, 65, pp. 496-513.

"Approche expérimentale des problèmes intéressant la production de la phrase noyau et ses constituants immédiats", avec L. Irigaray, Langages, 1966, 3, pp. 90-125.

"La neurolinguistique", Langages, 1967, 5, pp. 6-17.

"Description et classification des aphasies", Langages, 1967, 5, pp. 18-36

"La pathologie du langage", avec H. Hécaen, Revue de l'Enseignement supérieur, 1967, I, pp.

"Aspects des troubles de la vigilance au cours des lésions temporales antéro-internes droite et gauche", avec H. Hécaen et P. Marcie, Neuropsychologia, 1967, 5, pp. 311-328.

"Essai d'analyse neurolinguistique des agraphies", dans Livre d'hommage à Roman Jakobson, La Haye, Mouton, 1968.

"Description et classification des aphasies", avec P. Marcie et H. Hécaen, Langages, mars 1967, pp. 18-36.

"La désorganisation de la réception des signes verbaux dans l'aphasie sensorielle", avec H. Hécaen et P. Marcie, Revue d'Acoustique, 1968, 3-4, pp. 207-304.

"Mécanismes de l'aphasie et apports de la neurolinguistique à l'aphasiologie. Critères neurolinguistiques d'une classification des aphasies", avec H. Hécaen et P.Marcie, Acta neurologica et psychiatrica, 1968, 67, pp. 959-987.

"L'agraphie "pure", avec H. Hécaen et P. Marcie, Neuropsychologia, 1969, 7, pp. 271-286.

"Analyse linguistique d'énoncés d'aphasiques sensoriels", avec H. Hécaen, P. Marcie, M. Daumas, B. Lervielle-Anger, Journal de Psychologie, 1970, 2, pp. 185-206.

"La neurolinguistique", avec H. Hécaen, dans Applications of linguistics, Selected papers of the second International Congress of Applied Linguistics, Cambridge, C.U.P., 1971 pp. 85-99.

"Neurolinguistic Study of induction Aphasia", avec H. Hécaen, R. Angelergues, A. Maufras du Chatelier, P. Marcie, dans Psycholinguics and Aphasia, ed. H. Goodglass and S. 
Blumstein, 1972, Baltimore and London, The Jonhn Hopkins University Press, pp. 283-300

"De la linguistique à la neurolinguistique", Langages, 1977, 47, 3-38.

"L'agraphie des aphasiques sensoriels : les troubles à la dictée des mots et des logatomes", Langages, 1977, 47, 86-119.

\section{Derniers travaux publiés}

Dictionnaire étymologique et historique du français, avec F. Dubois-Charlier \& H. Mitterand. 2005. Refonte totale du Nouveau Dictionnaire étymologique. Larousse : Paris.

Le nombre en français, avec F. Dubois-Charlier, Aix, 2006, 207 pages. Repris dans la collection Poiein dirigée par A. Balibar, Amiens, E. M. E., 2008, 292 pages.

115 "La combinatoire lexico-syntaxique dans le Dictionnaire électronique des mots. Les termes de musique à titre d'illustration", avec F. Dubois-Charlier. 2010, Langages 179-180, pp. 31-56.

\section{Références d'accès aux dictionnaires informatisés de Jean Dubois et Françoise Dubois-Charlier}

\section{a) Ressources disponibles sur le site de MoDyCo (Université Paris Nanterre)}

116 https://www.modyco.fr/fr/base-documentaire/ressources/jean-dubois.html

117 Dictionnaire électronique des verbes français («LVF»), avec F. Dubois-Charlier, 1993, remanié en 1994 [2009], 25.610 entrées. Tableau Excel.

118 Les Verbes Français, avec F. Dubois-Charlier, 1993 [2009], transcription de la version électronique de 1994 [2009] de LVF. Document PDF.

119 Dictionnaire électronique des verbes français («LVF+1»), avec F. Dubois-Charlier, 2009. Version revue et corrigée de «LVF » par Paul Sabatier, sous la supervision des auteurs. Tableau Excel.

120 Dictionnaire électronique des mots («DEM »), avec F. Dubois-Charlier, 2015. Ouvrage posthume inachevét, près de 150000 entrées. Tableau Excel.

\section{b) Versions modernisées des dictionnaires informatisés de Dubois \& Dubois-Charlier, sur le site de l'IRO (Université de Montréal)}

http://rali.iro.umontreal.ca/rali/?q=fr/versions-informatisees-lvf-dem Interface de consultation groupée de LVF+1 et du DEM. Format JSON. Version procurée par Guy Lapalme.

123 Le site renvoie également à des versions antérieures en XML de LVF+1 et du DEM, ainsi qu'aux versions-sources en format Excel. 


\section{NOTES}

1. Cf. Il s'agit d'une version primitive de $L V F+1$ (cf. infra Titre IV).

2. Il s'agit d'une version primitive (perdue) du Dictionnaire Electronique des Mots (DEM), cf. infra Titre IV.

3. Ce travail semble avoir été perdu. En tout état de cause, les données ont été intégrées au Dictionnaire Electronique des Mots (DEM).

4. L'autorisation de publier le DEM a été donnée à Guy Lapalme et Denis Le Pesant par Françoise Dubois-Charlier quelques mois avant sa mort intervenue en 2016. Elle l'a fait avec réticence, vu le caractère inachevé de ce travail colossal. 